\title{
BOUNDED SOLUTIONS OF WHOLE-LINE DIFFERENTIAL EQUATIONS
}

\author{
BY DAVID LOWELL LOVELADY \\ Communicated by Fred Brauer, December 18, 1972
}

Let $Y$ be a finite-dimensional linear space with norm | |, and let $R=(-\infty, \infty)$. Let $\mathscr{A}$ be the algebra of linear functions from $Y$ to $Y$ with induced norm \|\| , and let $A$ be a locally integrable function from $R$ to $\mathscr{A}$. W. A. Coppel [2], [3, Theorem 1, p. 131] has determined necessary and sufficient conditions for

$$
u^{\prime}(t)=f(t)+A(t) u(t)
$$

to have at least one solution $u$ in $\mathscr{L}^{\infty}\left[R^{+}, Y\right]$ (where $R^{+}=[0, \infty)$ ) for each $f$ in $\mathscr{L}^{\infty}\left[R^{+}, Y\right]$. R. Conti [1] has solved the same problem for $f$ in $\mathscr{L}^{p}\left[R^{+}, Y\right], p \geqq 1$, and $u$ in $\mathscr{L}^{\infty}\left[R^{+}, Y\right]$. In this note we indicate how the results of [4] solve both of these problems for equations on $R$ instead of $R^{+}$. Let $\Phi$ be the fundamental solution for

$$
v^{\prime}(t)=A(t) v(t)
$$

i.e., $\Phi$ is that locally absolutely continuous function from $R$ to $\mathscr{A}$ such that $\Phi(t)=I+\int_{0}^{t} A(s) \Phi(s) d s$ whenever $t$ is in $R$.

THEOREM. Statements (i) and (ii) are equivalent and statements (iii) and (iv) are equivalent.

(i) Iff is in $\mathscr{L}^{\infty}[R, Y]$ there is a solution $u$ of $(\mathrm{NH})$ in $\mathscr{L}^{\infty}[R, Y]$.

(ii) There are three supplementary projections $P_{-1}, P_{0}$, and $P_{1}$ and a number $K$ such that if $t$ is in $R$ then

$$
\begin{aligned}
\int_{-\infty}^{t}\left\|\Phi(t) P_{-1} \Phi(s)^{-1}\right\| d s & +\left|\int_{0}^{t}\left\|\Phi(t) P_{0} \Phi(s)^{-1}\right\| d s\right| \\
& +\int_{t}^{\infty}\left\|\Phi(t) P_{1} \Phi(s)^{-1}\right\| d s \leqq K .
\end{aligned}
$$

(iii) Iff is in $\mathscr{L}^{p}[R, Y], p>1$, there is a solution $u$ of $(\mathrm{NH})$ in $\mathscr{L}^{\infty}[R, Y]$. (iv) There are $P_{-1}, P_{0}, P_{1}$, and $K$ as in (ii) such that if $t$ is in $R$ then

AMS (MOS) subject classifications (1970). Primary 34A30, 34E99. 


$$
\begin{aligned}
\int_{-\infty}^{t}\left\|\Phi(t) P_{-1} \Phi(s)^{-1}\right\|^{q} d s & +\left|\int_{0}^{t}\left\|\Phi(t) P_{0} \Phi(s)^{-1}\right\|^{q} d s\right| \\
& +\int_{t}^{\infty}\left\|\Phi(t) P_{1} \Phi(s)^{-1}\right\|^{q} d s \leqq K^{q},
\end{aligned}
$$

where $p+q=p q$.

If, in (iii), we take $p=1$, then (iv) can be replaced by (iv)* and we still have equivalence.

(iv)*

$$
\begin{aligned}
& \left\|\Phi(t) P_{-1} \Phi(s)^{-1}\right\| \leqq K \quad \text { if } t \geqq s, \\
& \left\|\Phi(t) P_{0} \Phi(s)^{-1}\right\| \leqq K \quad \text { for all }(t, s) \text { in } R \times R \text {, and } \\
& \left\|\Phi(t) P_{1} \Phi(s)^{-1}\right\| \leqq K \quad \text { if } t \leqq s .
\end{aligned}
$$

The implications (ii) $\rightarrow$ (i) and (iv) $\rightarrow$ (iii) are both obvious. Let $M_{0}$ be the subspace of $Y$ consisting of all values at zero of bounded solutions of (H). Let $M_{-1}$ be a subspace such that $M_{-1} \oplus M_{0}$ is all values at zero of solutions of $(\mathrm{H})$ bounded on $R^{+}$, and let $M_{1}$ be similarly determined by $R^{-}=(-\infty, 0]$. Let $M_{\infty}$ be determined by $Y=M_{0} \oplus M_{-1} \oplus M_{1} \oplus M_{\infty}$. Let $P_{0}, P_{-1}, P_{1}$, and $P_{\infty}$ be the projections of $Y$ onto $M_{0}, M_{-1}, M_{1}$, and $M_{\infty}$ respectively. If (i) holds then [4, Theorem 1] tells us that $P_{\infty}=0$, so (ii) can now be proved with standard techniques (see [3, pp. 131-134]). Similarly, if (iii) holds, the integral

$$
\int_{-\infty}^{\infty}\left\|P_{\infty} \Phi(s)^{-1}\right\|^{q} d s
$$

exists, and the linear function $\Lambda$ from $\mathscr{L}^{p}[R, Y]$ to $Y$ given by

$$
\Lambda[f]=\int_{-\infty}^{\infty} P_{\infty} \Phi(s)^{-1} f(s) d s
$$

is identically zero, so $P_{\infty}=0$. Now arguments similar to those of [1] can be used to establish (iii) $\rightarrow$ (iv).

\section{REFERENCES}

1. R. Conti, On the boundedness of solutions of ordinary differential equations, Funkcial. Ekvac. 9 (1966), 23-26. MR 37 \# 3102.

2. W. A. Coppel, On the stability of ordinary differential equations, J. London Math. Soc. 39 (1964), 255-260. MR 29 \#1393.

3. ㄴ Stability and asymptotic behavior of differential equations, D. C. Heath \& Co., Boston, 1965. MR 32 \#7875.

4. D. L. Lovelady, Boundedness and ordinary differential equations on the real line, J. London Math. Soc. (to appear).

Department of Mathematics, Florida State University, Tallahassee, Florida 32306 